\title{
Los servicios en la Gestión de Calidad
}

\section{Services on Quality Management}

*Aida Maribel Palma León MSc. ${ }^{1}$, **Fabiola Palma León², ***Rosa Torres Briones ${ }^{3}$, ****Karina Murillo Limones ${ }^{1}$

${ }^{1}$ Universidad Técnica Estatal de Quevedo, Facultad de Ciencias Empresariales, Escuela de Contabilidad y Auditoría. Campus Ing. Manuel Haz Álvarez, km 1.5 vía Santo Domingo de Los Tsáchilas. EC.120501. Quevedo, Ecuador

${ }^{2}$ Instituto de Seguridad Social

${ }^{3}$ Universidad Técnica de Cotopaxi

*apalma@uteq.edu.ec,**fapalma@uteq.edu.ec,***rosa.torres@utec.edu.ec, ****karina@hotmail.com

Fecha de recepción: $25 / 06 / 2018$

Fecha de aceptación: 25/10/2018

Publicado: 26/12/2018

\section{Resumen}

Este artículo ostenta que en la actualidad la gestión de calidad determina el crecimiento y mejora de las empresas, puesto a que para poder estar presente en un mercado cada vez más competitivo, las empresas tienen que apostar por la calidad del servicio. De tal manera, la implantación de una estructura operacional de trabajo bien documentada e integrada a los procedimientos técnicos y gerenciales, servirán de base para guiar las acciones de la fuerza de trabajo, los materiales y equipos, y por supuesto la atención que se debe dar a los consumidores. Este trabajo se orienta al estudio de los servicios en la gestión de calidad, el cual permite comprender de dónde proviene la necesidad de ofrecer un mejor servicio al cliente y en definitiva, a la sociedad, y de cómo se ha ido incluyendo toda la organización en la consecución de este fin. Este estudio que conlleva la partida sobre lo importante de la gestión de la prestación de servicios lo cual respalda esta tarea investigativa, para finalmente plantear las conclusiones propias de este cometido 
Palabras Clave: Servicios - gestión - calidad - Calidad de los servicios, mejora continua.

\begin{abstract}
This article holds that today quality management determines the growth and improvement of businesses, put that to be present in an increasingly competitive market, companies need to focus on quality of service. Thus, the implementation of a welldocumented and integrated technical and managerial procedures, operational working structure will provide the basis for guiding the actions of the labor, materials and equipment, and of course the attention to be given consumers. This work is oriented to the study of services in quality management, which allows us to understand where the need to offer a better service to the client and ultimately to society comes from, and how it has been including the entire organization in the attainment of this end. This study involves the departure on the importance of the management of the provision of services which supports this investigative task, to finally raise the conclusions of this assignment.
\end{abstract}

Keywords: Services, Management, Quality, Services, Continuous Improvement Quality.

\title{
Introducción
}

Actualmente, la calidad se manifiesta, y es reconocida, como factor imprescindible para la continuidad de las empresas en los mercados cada vez más competitivos. En términos simples se dice que un producto o servicio es de Calidad, cuando cumple las Expectativas del cliente, pero en la práctica, la Calidad, es algo más; es lo que sitúa a una empresa por encima o por debajo de los competidores, y lo que hace que, a medio o largo plazo, la empresa progrese o se quede obsoleta. 
Por otra parte Miranda Francisco, alega "Los importantes cambios que se han producido en el entorno competitivo durante los últimos años han llevado a las empresas a buscar soluciones para garantizar su supervivencia y crecimiento, respondiendo a las nuevas exigencias del mercado. Una de las respuestas que mejores resultados ha proporcionado es la implantación de una dirección basada en la calidad".

En el entorno actual no hay empresas viables sin atención al cliente y sin la calidad del servicio que se debe ofrecer. Por ello, para poder estar presente en un mercado cada vez más competitivo, las empresas tienen que apostar por la calidad del servicio.

Por lo mismo, esta figura expresa la calidad del servicio que el cliente espera recibir, primordialmente otorgando una excelente prestación, puesto a que cada vez los consumidores son más exigentes desde el bien que esperan recibir y de la forma que esperan que sea entregada, cumpliendo con sus expectativas tanto cuantitativa como cualitativamente. Dado a que el objetivo principal de toda organización es la satisfacción del cliente, y las exigencias determinan su crecimiento y constantes mejoras.

Miranda Francisco hace mención "Las empresas necesitan gestionar de forma eficaz no solo la calidad de sus productos propiamente dicha, sino también sus impactos en el medio ambiente y la prevención de riesgos laborales, tanto para ser competitivas, como para satisfacer los requisitos legales y las demandas cada vez más exigentes de la sociedad".

Ofrecer un servicio, fundamenta a su vez la ética que debe poseer la organización brindando transparencia y sinceridad. No se debe jamás prometer algo en falso, porque una promesa incumplida es un atentado a la credibilidad y a la verdad y conllevaría a que los clientes esperen mentiras y violaciones a su dignidad. 


\section{Los servicios y los cambios permanentes}

La actividad de servicio está sometida a cambios permanentes a los que la empresa debe hacer desde una perspectiva cada vez más profesional, Rodríguez afirma que "en la actualidad, para todas las empresas dedicadas a los servicios, lograr la plena "satisfacción del cliente" es un requisito más que indispensable para ganarse y posicionarse un lugar en la preferencia de los clientes... Por ello, el objetivo de mantener contento y satisfecho a cada cliente, es una tarea de toda la organización y principales objetivos de las empresas exitosas” Pág. 13.

La calidad en el proceso de prestación de los servicios está condicionada por pequeños detalles que, a través de los momentos de verdad, los clientes perciben de forma positiva o negativa. La percepción más o menos del momento de verdad puede estar afectada de forma directa por el contacto cliente-prestatario, es decir, una buena atención al cliente puede revertirse en satisfacción, fidelización, calidad e incluso en una estrategia que proporciona el servicio valor añadido y lo haría mucho más competitivo. "La calidad percibida es la variable que presenta limites menos claros respecto al concepto de satisfacción, ya que ambas se refieren a un proceso de evaluación en el que el cliente compara la experiencia dl servicio con determinadas expectativas previas, es decir, ambas tienen en común el hecho de que se considera central el punto de vista de los clientes, al valorar los servicios que presta una organización. (Bitner, 1990; Bolton y Drew, 1991; Parasuraman et al., 1988; Zeithaml et al., 1990).”

En el proceso actual de globalización económica, contar con un Sistema de Gestión Calidad, es un factor importante para la supervivencia y competitividad de las empresas y como primer paso para su implementación está el diagnóstico de la organización, según las Normas ISO-9001:2015 “Los Sistemas de Gestión de la 
Calidad, representan una herramienta de apoyo para garantizar la calidad en los servicios y, a su vez, la satisfacción del cliente ya que sus necesidades y las normativas de desempeño son complicadas de medir e identificar. Cada cliente es único, se define y se interpreta de acuerdo a sus propios criterios respetando su individualidad."

Asegurar servicios públicos de calidad adecuada para satisfacer necesidades de la colectividad que requiera definiciones que no han sido usuales en el sector público en la ciudad de Quevedo. "La calidad ha sido un aspecto olvidado de los servicios públicos porque ella es una dimensión menos cuantificable que los precios, más difícil de regular y menos tangible, pero los usuarios dan cuenta cuando falta (Palacios, 2000). Frente a esta situación, dos tendencias mundiales han aumentado el énfasis en la calidad de los servicios públicos en los últimos años: regulaciones más completas y estrategias gerenciales de administración de la calidad total (Simpson, 1998).”

Hoy existen ciudadanos mejor informados y consumidores inteligentes, conscientes de sus derechos, con más instancias de participación y con más poder para fiscalizar la acción pública. La cultura de la calidad y el servicio al cliente es un imperativo para poder atender las exigentes demandas y crecientes necesidades ciudadanas. Según Daniel Tigani El descuido del concepto de Excelencia en épocas de rápida evolución como esta, hace que el riesgo de perder nivel sea permanente, por eso la satisfacción de las demandas del cliente y la mejora continua son pilares de cualquier plan para obtener ventajas competitivas sostenibles. Así mismo Tigani afirma que El servicio es excelente, solo cuando hace crecer las expectativas de valor del cliente, de tal forma que para poder sorprenderlo, se tiene que superar a sí mismo continuamente. Pág. 30

El diagnóstico es indispensable para organizar, dirigir y proyectar todo el proceso de diagnóstico integral. La mejor forma de iniciar la implantación de un modelo 
de calidad es realizando una evaluación integral para tener un diagnóstico que permita conocer su situación actual, sus fortalezas, oportunidades, debilidades y amenazas y con base en el resultado, establecer estrategias y mecanismos para facilitar la implantación del modelo de calidad. Según los Sistemas de Gestión de la Calidad según ISO 9001:2000, Una vez que la organización ha decidido implantar un sistema de gestión de la calidad (SGC) o mejorar uno ya existente, debe analizar en qué medida su funcionamiento cotidiano se ajusta a los requisitos de la norma, para conocer la distancia que hay entre la gestión actual de la organización y el modelo de gestión propuesto por ISO 9001:2000.

La mayoría de las Instituciones de la localidad no cuentan con un Sistema de Gestión de Calidad y en la actualidad este particular está adquiriendo gran importancia en el medio, la Norma ISO 9001:2015 hace referencia que "Las empresas de servicios se ven enfrentadas a necesidades de cambios crecientes y a la adecuación de sus Sistemas de Gestión, como base y producto de la apertura de nuevos mercados, la intensificación de la competencia, la integración a la economía mundial y el rápido desarrollo de la tecnología...,en este panorama de alta competencia, la necesidad de las empresas de servicios basadas en procesos de mejoramiento y gestión de la calidad, se convierte en un elemento fundamental para alcanzar el éxito.”

El concepto de calidad, como tantos otros términos, ha variado a lo largo del tiempo y varía según el contexto en el que sea utilizado. En términos simples se dice que un producto o servicios es de Calidad, cuando cumple las Expectativas del cliente, pero en la práctica, la Calidad, es algo más; es lo que sitúa a una empresa por encima o por debajo de los competidores, y lo que hace que, a medio o largo plazo, la empresa progrese o se quede obsoleta, Duque hace referencia que "La calidad se enfoca a la prestación del servicio y depende de si el servicio ha cubierto o no las necesidades y las 
expectativas del cliente. La calidad se presenta hoy como sinónimo de buena gestión empresarial, lo que se traduce en productos y servicios competitivos”. Pág. 68

Esta evaluación nos ayuda a comprender de dónde proviene la necesidad de ofrecer un mejor servicio al cliente y en definitiva, a la sociedad, y cómo poco a poco se ha ido involucrando toda la organización en la consecución de este fin. La Norma ISO 9001:2000 describe "un Sistema de Gestión de la Calidad aplicable genéricamente a todas las organizaciones, sin importar su tipo, tamaño o su personalidad jurídica, dando prioridad a los clientes y tratando de ser compatibles con otros sistemas de gestión empresarial, como el Medioambiental y el de Prevención de Riesgos Laborales. La Gestión de la Calidad se ha convertido, por tanto, no ya en una alternativa a implantar por la empresa, sino una exigencia indispensable para la supervivencia de la misma y su posible competitividad en los mercados actuales"

\section{Características de los servicios}

Los servicios tienen las siguientes características elementales que los definen y diferencian del resto de los procesos. Daniel Tigani define en su libro de Excelencia de Servicio cuatro niveles que representan el efecto percibido por el cliente.

Básico: Son los atributos mínimos, sin ellos no tiene sentido alguno entrar en competencia. Sin embargo existen empresas en este nivel cuando gozan de privilegios y se forma un monopolio u oligopolio.

Esperado: Son los atributos que el cliente está seguro de recibir.

Deseado: Son los atributos que el cliente no necesariamente espera pero conoce y aprecia. 
Imprevisto: Son los atributos excepcionales que agregan valor sorpresa para el cliente, una vez que el cliente los conozca, se convertirán en deseados. Es en este nivel donde comienza la excelencia. Pág. 46

Los servicios son intangibles ya que no se les puede ver, probar, palpar oír ni oler antes de adquirirlos. En relación con esta característica, se expresa que el elemento fundamental no es el producto físico, lo cual resulta una mejor forma de definirla. La mayoría de las empresas de servicios entregan productos no tangibles, otras suministran un elemento tangible pero sólo incidentalmente forma parte del servicio suministrado al cliente. Una de las características principales de los servicios que expresa Lara es la "Intangibilidad", esta es la característica principal que distingue a los servicios de los productos, es que no pueden poseerse físicamente. La intangibilidad es la cualidad de no ser percibido por medio de los sentidos de la vista, del oído, del gusto, del tacto o del olfato. Pág. 3

Además debemos de considerar que los servicios no son almacenables, ni transportables. Estos se pueden suministrar a los clientes, sólo en el momento que son creados, no pueden ser medidos en términos físicos ni verificados antes de ser vendidos. Esto se asemeja a lo dice Lara en las características principales de los servicios. La Imperdurabilidad es el resultado de la característica anterior; es decir, que la capacidad de servicio que no se utiliza, no puede ser almacenada para su uso posterior y Simultaneidad entre producción y consumo nos dice que en los servicios, la producción y el consumo son simultáneos. Si no hay usuario no hay servicio.

Un servicio es inseparable de su fuente, trátese de una persona o máquina, en él coinciden el tiempo de producción y el tiempo de consumo, el elemento de entrada, que se transforma en objeto de salida es una persona. Sánchez E. y Pla J. enuncian "La 
inseparabilidad de los servicios y el grado de adaptación a las necesidades del cliente como dimensiones clave para agrupar lo servicios debido a las importantes repercusiones que éstas tienen en un contexto internacional”. Pág. 31

Los servicios son sumamente variables, pues dependen de quienes los suministran y del momento y lugar en que se llevan a cabo. Según Cottle, David "El servicio constituye un elemento central en la mayoría de los productos tangibles. Todos los productos presentan tanto características tangibles como intangibles. Un servicio más que un producto es un proceso esto significa que la actividad o proceso es parte de la producción de un servicio, tanto como lo es el producto final de ese servicio"

Existen varias razones por las que se considera importante la calidad del servicio, una de ellas y la más importante es que los clientes son cada vez más críticos respecto a los servicios que reciben. Muchos clientes, no sólo desean un servicio mejor sino que lo esperan. Otra de las razones es que el crecimiento nuevo del futuro va a concentrarse más aún sobre el sector de servicios. Alén y Fraiz afirman que "La calidad percibida es la variable que presenta limites menos claros respecto al concepto de la satisfacción, ya que ambas se refieren a un proceso de evaluación en el que el cliente compara la experiencia del servicio con determinadas expectativas previas, es decir, ambas tiene en común el hecho de que considera central el punto de vista de los clientes, al valorar los servicios que presta una organización.” Pág. 252

\section{Calidad en los servicios}

La calidad del servicio es la percepción que tiene el cliente acerca de la correspondencia entre el desempeño y las expectativas, relacionada con el conjunto de los elementos secundarios, cuantitativos y cualitativos, de un producto o servicio principal. En cuanto a la calidad del servicio, podemos inferir que sus dimensiones no 
son tan obvias con el peso, la longitud, la dureza, etc. Las mismas se deben tener en cuenta para medir la calidad del servicio, según Rincón "la calidad de servicio es cumplir con las expectativas que tiene el cliente sobre el bien o servicio que necesita el cliente, se considera importante la calidad del servicio debido que los clientes son cada vez más críticas respecto a los servicios que reciben” Pág. 9.

Todo lo que implica ser bien atendido, como por ejemplo ser bien recibido, sentirse apreciado, ser escuchado, recibir información, ser ayudado. No debemos dar lugar a la apatía, la indiferencia o el desprecio y debemos despojarnos de nuestros prejuicios y motivados por la impresión o apariencia que muestra el cliente. Según Botero "El cliente califica que tan competente es el empleado para atenderlo correctamente; si es cortes, si conoce las empresa donde trabaja y los productos o servicios que vende, si domina las condiciones de venta y las políticas, en fin, si es capaz de inspirar confianza con sus conocimientos como para que usted le pida orientación” Pág. 219.

Se debe establecer claramente que se está entendiendo bien al cliente y que también nosotros estamos siendo entendidos. El personal encargado de prestar el servicio no debe dejarse seducir por la jerga propia de determinada especialidad, ya que se puede estar en presencia de alguien que no entienda nada acerca de lo que se le está hablando. Resulta fatal esperar para que alguien nos comunique que está en el lugar equivocado o a la hora equivocada. Blum, G., Lozzia, G., Abal, F., Picón J., Attorresi H. manifiestan que "ninguna acción de ventas (por muy sencilla que sea) puede llevarse adelante si no se analizan previamente los complejos mecanismos del cerebro humano". Los psicólogos de empresa deben prestar atención a los componentes subjetivos puestos en juego en la interacción Vendedor-Cliente, que no sólo deberían facilitar la adquisición de un producto por parte del consumidor, sino que además éste 
quede convencido de que aquello por lo que ha pagado le va a ser útil, le proporciona un beneficio y le satisface alguna de sus necesidades... Indican también que "Cada vendedor es una empresa independiente con un solo empleado”. Pág. 118

\section{Satisfacción de los clientes}

Se debe generar capacidad para mostrar afecto por el cliente interno y externo. Se debe respetar la sensibilidad de la gente, porque muchas veces es altamente vulnerable a nuestro trato. Cuando se trata de atender reclamos, quejas y clientes irritables, no hay nada peor que una actitud simétrica o de mala voluntad, por lo que ser amables resulta un elemento de vital importancia, Botero define que "el servicio al cliente es el conjunto de estrategias que una compañía diseña para satisfacer, mejor que sus competidores, las necesidades y expectativas de sus clientes externos”. Pág. 219

Nunca se debe mentir al cliente, porque después de una mentira, el cliente sólo puede esperar nuevas mentiras y violaciones a su dignidad. No se debe jamás prometer algo en falso, porque una promesa incumplida es un atentado a la credibilidad. Crear expectativas exageradas es falta de compromiso con el cliente y desprecio por la verdad. Tigani en su libro de Excelencia de Servicio Señala que la ambientación, el aspecto del personal, el lenguaje utilizado en la comunicación, etc. Son característicos del tipo de comercio y pueden agregar o destruir valor para el cliente. Nunca esta demás recordar, que un cliente insatisfecho, se convertirá en un cliente perdido, volcado a comprar productos de la competencia, que en su retirada puede arrastrar a otros clientes, bien sean actuales o potenciales. Pág. 38... además es importarte resaltar lo que menciona J. Refugio Lara López sobre la fiabilidad es importante debido a que representa, la habilidad que posee la empresa para suministrar el servicio prometido de forma fiable, 
segura y cuidadosa. Dar fiabilidad al cliente es también cumplir lo que se promete en folletos, hojas de horarios, etc. Pág. 4

Para elevar la calidad del servicio es necesario que los recursos que se utilizan también lleven calidad, hay que descubrir qué está ocurriendo en la mente del cliente y verificar las interacciones entre la institución y el cliente en todos los puntos de contacto. Todo esto de manera que el éxito o fracaso de las empresas dependen en gran parte de su capacidad para identificar los factores que son importantes para los clientes y para vigilar que la empresa funcione de manera competitiva con esos factores. Las percepciones de los consumidores sobre la calidad de servicio están directamente influidas por la di confirmación e indirectamente por la disconfirmación, las expectativas y el actual nivel de desempeño (vía satisfacción/insatisfacción). Además, mediante un efecto indirecto en la satisfacción vía disconfirmación, el desempeño percibido tiene un efecto directo sobre la satisfacción, particularmente en servicios de alta implicación; aunque también se ha encontrado evidencia del efecto directo en la CALIDAD de servicio percibida (Bolton y Drew,1991b; Cronin y Taylor, 1992).

Oliver y De Sarbo (1988) encuentran que tanto la disconformidad como la calidad percibida tienen un impacto más fuerte en la satisfacción que las expectativas.

La satisfacción está basada en la experiencia con el servicio, mientras que la calidad de servicio percibida no está basada necesariamente en la experiencia. Por ello, la investigación sobre satisfacción se ha centrado en evaluaciones post-consumo, mientras que la de calidad de servicio ha focalizado su atención en evaluaciones pre decisionales (Latour y Peat, 1979). Pág. 253.

Existen experiencias empresariales que evidencian el manejo de los términos: calidad y satisfacción como sinónimos, aunque algunos estudios de investigadores apuntan a marcar diferencias entre ambos términos. El enfoque más reciente del 
concepto de satisfacción del cliente, se basa en la satisfacción acumulada de todas las experiencias previas al consumo a lo largo del tiempo, representando el resultado de un proceso de aprendizaje en el que el consumidor aprende o recuerda su satisfacción, basándose en las transacciones anteriores. Caballero manifiesta esta concepción de "La satisfacción en el trabajo como una actitud se distingue básicamente de la motivación para trabajar en que ésta última se refiere a disposiciones de conducta, es decir, a la clase y selección de conducta, así como a su fuerza e intensidad, mientras que la satisfacción se concentra en los sentimientos afectivos frente al trabajo y a las consecuencias posibles que se derivan de él” Pág. 2.

La empresa tiene que afrontar ese continuo deseo de satisfacción que siente su cliente. Si el cliente no siente esa plena satisfacción en esa empresa, no dudará, más tarde o temprano, en ignorarla y marcharse a otra de la competencia, por tanto, en este sentido el cliente es despiadado con la empresa y si ésta no la satisface no conseguirá su fidelidad. Todo problema interno de la empresa que resienta la plena satisfacción del cliente, finalizará provocando la pérdida del mismo y ello se refleja, sin duda, en los resultados económicos empresariales. Según Lara J. Las organizaciones de servicio deben de determinar qué beneficios esperan recibir los clientes y de procurar producir los servicios que puedan colmar y si es posible exceder sus expectativas. La calidad del servicio es la conformidad del servicio prestado con las especificaciones y expectativas del cliente. Pág. 5.

Es la era de la CALIDAD, de la MEJORA CONTINUA y de la lucha y trabajo continuo por conseguir la EXCELENCIA. Por lo tanto, cuanto profunda y amplia sea esta invasión, más potente y COMPETITIVA, invasión, más potente y COMPETITIVA, según Fonte M, Guerrero G, Giráldez R "La calidad de la mejora continua es despertar nuevas necesidades del cliente, lograr productos y servicios de 
calidad, producir y entregar un producto al cliente de acuerdo a su necesidad y así mismo para que una empresa sea altamente competitiva debe obtener la satisfacción total de su clientela" Pág.5.

Es decir, la satisfacción del cliente es el objetivo primordial de toda Organización: si la empresa cuenta con clientes satisfechos es seguro que tendrá beneficios, ahora y en el futuro. Y por este motivo medir la satisfacción de los clientes es la mejor forma de saber si éstos están satisfechos o no con los servicios de la empresa, La Norma ISO 9001:2000 afirma que "La satisfacción del cliente es la percepción que el cliente tiene sobre el grado en que se han cumplido sus requisitos..., es un estado psicológico, y por tanto subjetivo, cuya obtención asegura fidelidad..., impulsa a las organizaciones a que alcancen la satisfacción del cliente.., como una de las medidas del desempeño del sistema de gestión de la calidad, la organización debe realizar el seguimiento de la información relativa a la percepción del cliente respecto al cumplimiento de sus requisitos por parte de la organización".

La satisfacción del cliente se puede definir como la diferencia entre las expectativas y la percepción del cliente respecto al producto o servicio ofrecido.

Es decir, un cliente está satisfecho cuando su percepción del producto o servicios coincide con o supera sus expectativas. Duque expresa que "es el conjunto de prestaciones que el cliente espera, además del producto o servicio básico, como consecuencia del precio, la imagen y la reputación del mismo” Pág. 65.

Las principales razones por las que se da una diferencia entre lo que la empresa ofrece y lo que percibe el cliente son las siguientes:

1. La empresa puede que no tenga una orientación hacia el cliente y define sus productos o servicios de acuerdo con sus opiniones y no las del cliente. 
2. Asimismo, no hay muchas empresas que se pongan sistemáticamente en lugar del cliente para tener una idea clara de su satisfacción.

3. La capacidad que tienen muchas empresas de pensar que sus productos son excelentes y que es imposible que el cliente pueda estar insatisfecho.

Cottle, David Manifiesta: "Los profesionales no tienen relación tan simple con sus clientes. Debido que la producción y consumo de los servicios constituyen fases simultaneas ambas se mezclan entre sí y también se mezclan con las actividades de marketing"

\section{Conclusiones}

La satisfacción del cliente debe abordarse con subjetividad, pero teniendo en cuenta que es la subjetividad del cliente y no la de la empresa, porque de nada vale realizar un producto técnicamente perfecto, si no satisface la necesidades y expectativas de los clientes, en resumen, si a nadie le interesa.

Guiamet J. manifiesta que los clientes "son las personas más importantes para cualquier negocio"; "no dependen de nosotros sino que nosotros dependemos de ellos", ya que "nos hacen un favor al comprar nuestros productos. Pág. 365

Comprender y reconocer al cliente implica esforzarse en conocer sus necesidades y sobre todo: aprender los requerimientos específicos del cliente, ya que la mejor forma de conocer su necesidades es hablando con él y transmitirle que para la empresa "el cliente es lo primero", Peñaloza sostiene en términos muy sencillos "que el hombre busca experiencias gratificantes que le aporten utilidad y beneficios,

y justamente es lo que procura la mercadotecnia al estudiar y comprender las 
necesidades y deseos del consumidor, y ofertar un mix ajustado a los gustos de los compradores, entregando beneficios y agregando valor” Pág. 73.

Reconocer al cliente habitual, que éste suele representar la base del negocio actual de la empresa. Esforzase en que cada persona de la empresa sepa quién es el cliente y cómo pueden contribuir a satisfacer sus necesidades, según Najul cuando se trata de satisfacer al cliente, todos somos un equipo, todas las personas de la organización deben estar alineadas a satisfacer al cliente. Pág. 27

\section{Referencias Bibliográficas}

Alén Gonzalez, M. E.; Fraiz Brea J. A. Relación entre la Calidad del Servicio y la Satisfacción del Consumidor. Su Evaluación en el Ámbito del Turismo Termal. Universidad de Vigo. (Pág. 252, 253). Aedem-Virtual [Revista en Internet]. 2006 Ene. [Citado 2015 Ago 12]; Disponible en: http://www.aedemvirtual.com/articulos/iedee/v12/121251.pdf

Blum, G. Diego; Lozzia, Gabriela; Abal, Facundo; Picón Janeiro, Jimena; Attorresi, Horacio. Factores de personalidad predictores de la disposición para la venta. Un estudio exploratorio con postulantes laborales. Facultad de Psicología. Universidad de Buenos Aires. (Pág. 118). SCIELO [Revista en internet]. EneDic 2009 [Citado 2015 Ago. 03]; Disponible en: http://www.scielo.org.ar/pdf/anuinv/v16/v16a11.pdf

Botero María Mercedes, Peña Paola. Calidad en el servicio: El cliente Incógnito. Universidad del Norte. Barranquilla, Colombia. (Pág. 219). REDALYC [revista en internet]. 2006 Sept. [Citado 2015 Ago 02]; Disponible en: $\underline{\text { http://www.redalyc.org/pdf/1342/134216870007.pdf }}$

Caballero Rodríguez Katia. El concepto de "satisfacción en el trabajo" y su proyección en la enseñanza. (Pág. 2). Currículum y Formación del profesorado [Revista en internet]. 2002 [Citado 2015 Ago 02]; Disponible en: http://www.ugr.es/ recfpro/rev61COL5.pdf 
Cottle David. El Servicio Centrado en el Cliente. Ediciones Díaz de Santo S.A. (Pág. 61, 63). Madrid-España. [Sitio de internet]. . Disponible en: https://books.google.com.ec/books?hl=es\&lr=\&id=o37JeJi0qoC\&oi=fnd\&pg=PA37\&dq=diferencias+entre+la+empresa+y+clientes\&ots $=4$ HWMTDveY\&sig=tKLhb9gm-

HxbkP4j5vNGeHwE5A4\#v=onepage\&q=diferencias\%20entre\%20la\%20empres a\%20y\%20clientes\&f=false

Daniel Tigani. Excelencia en Servicio $1^{\circ}$ Edición. (Pág. 30, 38, 45, 46,) [Sitio de internet]. [Citado 2006]; liderazgo 21. Disponible en: http://capacitacion.agama.com.mx/agamacapacitacion/Libros/Libro\%20de\%20 Ventas.pdf

Duque Oliva Edison Jair. Revisión del concepto de calidad del servicio y sus modelos de medición. (Pág. 68,70). Innovar [Revista en internet]. 2005 Ene-Jun [Citado 2015 Ago 02]; Disponible en: http://www.redalyc.org/articulo.oa?id=81802505

Duque Oliva, Edison Jair. Revisión del concepto de calidad del servicio y sus modelos de medición. (Pág. 65, 68, 70). INNOVAR [revista en internet]. 2005 Junio [Citado 2015 Ago 02]; Disponible http://www.redalyc.org/articulo.oa?id=81802505

Fonte Marianelys, Msc. Guerrero Gisela, Tlga. Giraldez Raisa. Diagnóstico y evaluación de la calidad de los servicios en la Biblioteca de la Universidad de Matanzas "Camilo Cienfuegos". (Pág.5). Biblioteca Universidad de Matanzas [Sitio de internet]. Citado 2015 Ago 02; Disponible en: http://www.bibliociencias.cu/gsdl/collect/eventos/index/assoc/HASHbe58.dir/do c.pdf

Guiamet Jaime. El cliente siempre tiene la razón: Procesos de construcción de subjetividad en trabajadores jóvenes de una cadena multinacional de supermercados en la ciudad de Rosario. (Pág. 365). Caicyt-Cocinet [Revista en internet]. 2012 Dic [Citado 2015 Ago 02]; Disponible en: http://www.unse.edu.ar/trabajoysociedad/19\%20GUIAMET\%20subjetividad\%2 0Trabadores\%20jovenes\%20Rosario.pdf 
Hernández Rodríguez Carlos. El servicio al cliente como factor de competitividad y calidad en las empresas de servicios: Caso de las organizaciones escolares particulares. (Pág. 13). IIESCA [revista en internet]. 2014 May [Citado 2015 Ago 02]; Disponible en: http://www.uv.mx/iiesca/files/2014/09/02CA201401.pdf

Indicaciones Para Complementar El Cuestionario De Diagnóstico Previo, Sistemas de Gestión de la Calidad según ISO 9001:2000. (Pág 1) [Sitio en internet]. Disponible en: http://www.fundacioncetmo.org/DGT\%20Mejora\%20Continua/pdf/Anexos/I/IA \%20-\%2011.pdf

Lara, López, J. Refugio. La Gestión de la Calidad en los servicios. Conciencia Tecnológica, núm. 19, abril, 2002, p. 0 Departamento de Ingeniería Industrial Instituto Tecnológico de Aguascalientes. (Pág. 3, 4, 5) [Revista en internet]. 2002 Abr [Citado: 2015 Ago 04] Disponible en: http://www.redalyc.org/articulo.oa?id=94401905

Miranda González Francisco Javier, Chamorro Mera Antonio, Rubio Lacoba Sergio. Introducción a la gestión de la calidad. DELTA. Primera Edición. 2007. MadridEspaña. Disponibles en: https://books.google.com.ec/books?hl=es\&lr=\&id=KYSMQQyQAbYC\&oi=fnd \&pg=PA1\&dq=introducciones+sobre+gestion+de+calidad\&ots=Isr9kflP6j\&sig =QrPsgtyBnLZfIlEwJMQoEChMKFw\#v=onepage \&q=introducciones\%20sobre \%20gestion

Montaña Rodríguez Joaquín; Ramírez Plaza Hernando. Evaluación de la calidad de los servicios públicos domiciliarios. Universidad Autónoma de Bucaramanga. Bucaramanga-Colombia. (Pág. ). REDALYC [revista en internet]. 2002 Dic. [Citado 2015 Ago. 12]; Disponible http://www.redalyc.org/articulo.oa?id=10900506

Najul Godoy Jenny. El Capital Humano en la atención al cliente y la calidad del servicio. Universidad Central de Venezuela. (Pág. 27). REDALYC [Revista en internet]. Jul-Dic 2011 [Citado 2015 Ago. 02]; 
Norma ISO 9001. La satisfacción del cliente. 2008 Marz0 [Citado 2015 Ago 02]

Normas ISO 9001:2015. Proceso de prestación del servicio. Escuela Europea de Excelencia [Blog en internet]. 2015 Ene 14 [Citado 2015 Ago 02]

Peñaloza Marlene. El Mix de Marketing: Una herramienta para servir al cliente. Actualidad Contable Faces. (Pág. 73). REDALYC [revista en internet]. 2005 Jun [Citado 2015 Ago 02]

Rincón Soto Idana. Inteligencia ética y calidad de servicio en las Instituciones de Educación Superior. (Pág. 9). Entelequia [revista en internet]. 2014 [Citado 2015 Ago 02

Sánchez Peinado Esther, Pla Barber José. La inseparabilidad y el grado de adaptación como determinantes de la internacionalización de las empresas de servicios. (Pág. 31). Roderic [Revista en internet]. 2005 Dic [Citado 2015 Ago 02]. 